\title{
Sicherung der Prozessqualität in der pneumologischen Praxis mittels Peer-Review-Verfahren - ein Pilotprojekt
}

\author{
Assurance of Process-Quality in Pulmonary Practice by Means of a \\ Peer-review System - a Pilot Project
}

Autoren

Institut
J. Fischer ${ }^{1}$, D. Köhler ${ }^{2}$, H. Magnussen ${ }^{3}$

Institut für Rehabilitationsforschung Norderney am Lehrstuhl für Klinische Rehabilitations-wissenschaften der Universität Witten/Herdecke (Direktor: Prof. Dr. J. Fischer),

Krankenhaus Kloster Grafschaft, Schmallenberg (Ärztlicher Direktor: Prof. Dr. D. Köhler),

Krankenhaus Großhansdorf, Zentrum für Pneumologie und Thoraxchirurgie (Ärztlicher Direktor: Prof. Dr. H. Magnussen) eingereicht 30.1. 2006

akzeptiert 24.4. 2006

\section{Bibliografie}

DOI $10.1055 / \mathrm{s}-2006-932212$

Pneumologie 2006; 60;

485-492

(c) Georg Thieme Verlag KG Stuttgart · New York .

ISSN 0934-8387

\section{Korrespondenzadresse}

Prof. Dr. Jürgen Fischer

Klinik Norderney der Deutschen Rentenversicherung Westfalen . Klinik der Universität Witten/ Herdecke

Kaiserstraße 26

26548 Norderney

fischer-norderney@t-online.de

\section{Zusammenfassung}

Die Qualitätssicherung und das Qualitätsmanagement sind in der sozialen Gesetzgebung seit Jahren verankert. Es wurden in pneumologischen Akut-Kliniken, Rehabilitationskliniken und schlafmedizinischen Zentren wiederholt PeerReview-Verfahren zur Sicherung der Prozessund Ergebnisqualität durchgeführt. Im Rahmen von einer Pilotstudie sollte geklärt werden, ob in der pneumologischen Praxis eine Sicherung der Prozessqualität möglich ist und ob ein Peer-Review-Verfahren hierfür die geeignete Methode darstellt. In 44 pneumologischen Fachpraxen wurden jeweils 9 Berichte von Patienten mit Asthma bronchiale oder COPD an den Hausarzt und/oder Untersuchungsprotokolle der eingesetzten Testverfahren, wie Lungenfunktion, Allergietest, bronchialer Hyperreagibilitätstest usw. zufällig ausgewählt und jeweils 3 Gutachtern (Peers) zugeleitet. Es führten 396 ausgewählte Patientendatensätze zu 1188 Begutachtungen. Mittels einer eigens erstellten Checkliste qualitätsrelevanter Prozessmerkmale und eines dazugehörigen Manuals wurde die Begutachtung von den Peers vorgenommen, Qualitätsmaßzahlen ermittelt und ein individueller Ergebnisbericht für jede einzelne Praxis erstellt. Zusätzlich wurde die gesundheitsökonomische Bewertung von Diagnostik und Therapie sowie die Beurteilung des Gesamtprozesses mittels visueller Analogskalen vorgenommen. Für deutlich mehr als die Hälfte der Beurteilungen konnten keine Mängel festgestellt werden. Bei etwa jeder 6. Beurteilung wurden jedoch für die verschiedenen Items erhebliche Mängel bzw. ein inakzeptables Ergebnis festgestellt. Für die einzelnen Items wurde ein grafisches Ranking im Sinne eines Benchmarking in dem individuellen Ergebnisbericht dargestellt. Die Peers, die Gutachter und Begutachtete gleichzeitig waren, können aufgrund der Erfahrungen, die sie bei der Begutachtung gewonnen

\section{Abstract}

Quality assurance and quality-management have been well anchored in social legislation for years. Peer-reviews to assure process-quality and quality of outcomes have been conducted repeatedly in centres for pulmonary medicine, facilities for pulmonary rehabilitation as well as centres for sleep-medicine. By means of a pilot study we wanted to investigate if assurance of processquality is feasible and if a peer review system turns out to be a suitable method in pulmonary practice. Medical reports and/or diagnostic protocols of the investigational procedures such as pulmonary function tests, allergy-tests, induction test of bronchial hyperresponsiveness et cetera were randomly collected from nine patients with bronchial asthma or COPD in each of the 44 participating pulmonary practices and forwarded to three peer-reviewers respectively. 396 selected patient-datasets resulted in 1188 reviews. Peer review was conducted by means of a specially developed checklist of markers of processquality accompanied by an explanatory manual. Measures of quality were determined for every pulmonary practice and a report of results was generated. The health economical efficacy of the therapy and the diagnostic process as well as an assessment of the overall process was evaluated by means of a visual analogue scale. No deficiencies could be detected in clearly more than half of the assessments. Major deficiencies or an unacceptable result in different items could be detected in every sixth assessment. For every item a graphic ranking in terms of benchmarking was generated for each individual report. Since peers were reviewers and subjects of review at the same time, they can now clearly improve and assure their diagnostic and therapeutic processquality in patients with bronchial asthma and COPD by means of the experience they gained during the review process, as well as by the as- 
haben und aufgrund ihres individuellen Ergebnisberichtes die Prozessqualität bei der Behandlung von Patienten mit Asthma bronchiale und COPD deutlich steigern und sichern. Es konnte gezeigt werden, dass ein Peer-Review-Verfahren zur Sicherung der Prozessqualität in der pneumologischen Praxis geeignet ist und es möglich ist, eine kontinuierliche Qualitätssicherung und -verbesserung auf Dauer zu erreichen. sessment they received. We were able to demonstrate that a peer-review-system is appropriate to assure process-quality in pulmonary practice and that it is feasible to achieve a continuous assurance and improvement of quality in the long run.

\section{Einleitung}

Das Interesse des Gesundheitswesens an der Qualitätssicherung und dem Qualitätsmanagement im Gesundheitswesen hat in den letzten Jahren zugenommen. Die begrenzten finanziellen Ressourcen führen dazu, dass nur noch solche Leistungen erbracht werden können, die dem medizinisch-wissenschaftlichen Erkenntnisstand entsprechen. Die gesetzliche Regelung zur Qualitätssicherung und zu ihrer Weiterentwicklung im Rahmen der Gesundheitsreform 2000 hat sich im SGB V in $§ 135$ a folgendermaßen niedergeschlagen: „Die Leistungserbringer sind zur Sicherung und Weiterentwicklung der Qualität der von ihnen erbrachten Leistungen verpflichtet. Die Leistungen müssen dem jeweiligen Stand der wissenschaftlichen Erkenntnisse entsprechen und in der fachlich gebotenen Qualität erbracht werden.“

In der Pneumologie hat die Qualitätssicherung einen hohen Stellenwert erreicht. Bereits 1993 erfolgte die erste Akkreditierung von schlafmedizinischen Zentren durch die Deutsche Gesellschaft für Schlafforschung und Schlafmedizin (DGSM) [1,3]. 1999 erfolgte die Sicherung der Prozessqualität und seit 2002 auch die Sicherung der Ergebnisqualität im Bereich der schlafmedizinischen Zentren der DGSM [2,4]. Seit 1994 wird im Auftrag des Verbands Deutscher Rentenversicherungsträger (VDR) ein Qualitätssicherungsprogramm zur Überprüfung der Prozessqualität durchgeführt [5]. In diesen Prozess sind auch die pneumologischen Rehabilitationskliniken eingebunden. Der Arbeitskreis pneumologischer Kliniken hat 1995 erstmals ein Projekt zur Qualitätskontrolle in pneumologischen Kliniken eingeführt. Patientenakten werden durch Oberärzte und Chefärzte in regelmäßigem Abstand begutachtet. Da Standards nicht direkt vorgeschrieben waren, wurde dieses Verfahren der Qualitätssicherung als evolutionäre Qualitätssicherung bezeichnet [6]. Es wird dabei durch den regelmäßigen, sich wiederholenden Beurteilungsprozess eine Verbesserung der Qualität und Weiterentwicklung der Begutachtung angestrebt.

Im Bereich der ambulanten Pneumologie erfolgt bisher keine Sicherung der Prozessqualität. Dies hat niedergelassene Pneumologen und Experten veranlasst, sich mit der Thematik auseinander zu setzen. Im Jahre 2003/04 wurde im Raum Hamburg/ Schleswig-Holstein ein Pilotprojekt durchgeführt, an dem 12 pneumologische Praxen erfolgreich teilnahmen. Es wurde eine Checkliste mit praxisrelevanten Merkmalen und ein Manual erarbeitet, welches die Qualitätsstandards definierte. 60 Inhaber von pneumologischen Praxen im norddeutschen Raum wurden eingeladen, das Pilotprojekt zur Sicherung der Prozessqualität in der pneumologischen Praxis vorgestellt sowie ein weiteres Pilotprojekt mit 44 Praxen geplant und durchgeführt. Ziel dieser beiden Pilotprojekte war es zu überprüfen, inwieweit eine Sicherung der Prozessqualität in der pneumologischen Facharztpraxis grundsätzlich möglich, erforderlich und sinnvoll ist und inwieweit hierzu ein Peer-Review-Verfahren eingesetzt werden kann.

\section{Methode}

In 44 pneumologischen Fachpraxen wurden zwischen dem 15.10. - 15.12.2004 alle neu in der jeweiligen Praxis behandelten Patienten mit der Arbeitsdiagnose Asthma bronchiale oder COPD gelistet und an die wissenschaftliche Projektstelle im Institut für Rehabilitationsforschung, Norderney am Lehrstuhl für Klinische Rehabilitationswissenschaften der Universität Witten/ Herdecke versandt. Mithilfe eines Zufallsgenerators wurden neun Akten pro Praxis ausgewählt. Die Akten wurden nach Anonymisierung dreimal kopiert und den nach dem Zufallsprinzip ausgewählten Gutachtern (peers) zugesandt. Jede Praxis wurde von drei Gutachtern bewertet. Jeder Gutachter erhielt somit 27 Akten, sodass aus 396 Patientendatensätzen 1188 Begutachtungen resultierten.

Die Begutachtung erfolgte anhand einer Checkliste von qualitätsrelevanten Prozessmerkmalen für die pneumologische Praxis. Die Checkliste hatte ein Steuerungskomitee aus fünf niedergelassenen Fachärzten entworfen. Die zeitgleiche Beurteilung der Prozesse erfolgte anhand eines Manuals, welches Leitlinien und Empfehlungen berücksichtigte. Zur Prozessbeurteilung wurden der Bericht an den überweisenden Arzt - in den meisten Fällen der Hausarzt - sowie funktionsanalytische Befunde und Untersuchungsprotokolle zur spirometrischen oder ganzkörperplethysmografischen Funktionsdiagnostik, Bronchospasmolysetests, Röntgenbildbeschreibungen, Allergietests, CO-Diffusionskapazitätsmessungen und Hyperreagibilitätstests herangezogen. Der Bericht an den Hausarzt wurde bezüglich Anamnese, Untersuchungsbefund, Diagnose und Therapie sowie der epikritischen Zusammenfassung beurteilt (Abb. 1). Die Beurteilung der einzelnen Prozessmerkmale erfolgte anhand einer LikertSkala nach dem Vorliegen von „keine Mängel“, „leichte Mängel“, „erhebliche Mängel“ und „inakzeptabel“ sowie „nicht beurteilbar“. Die Definition der verschiedenen Abstufungen war im Manual für die einzelnen Prozessmerkmale beschrieben. Falls Teile des Berichts oder Befunde mit „nicht beurteilbar“ klassifiziert wurden, wurde dieser Wert als fehlend, d.h. als „missing value“ gewertet. Die Checkliste wurde abgeschlossen mit drei visuellen Analogskalen (VAS) mit denen sowohl gesundheitsökonomische Aspekte von Diagnostik und Therapie als auch eine Beurteilung des Gesamtprozesses vorgenommen werden sollte (Abb. 2). Mit dieser visuellen Analogskala sollte beurteilt werden, ob der Gesamtprozess dem aktuellen Stand wissenschaftlicher Erkenntnisse, Empfehlungen und Leitlinien entspricht. Schließlich konnten die Gutachter auch schriftliche Kommentare zu den vorliegenden Befunden abgeben.

Die Auswertung der Begutachtung erfolgte in der wissenschaftlichen Projektstelle. Hier wurde für jede Praxis ein Bericht abgefasst, um den Praxen die Erkennung von Defiziten und die Einleitung von Verbesserungsmaßnahmen zu ermöglichen (Tab. 1). 


\section{Checkliste \\ qualitätsrelevanter Prozessmerkmale}

Abb. 1 Checkliste qualitätsrelevan-

ter Prozessmerkmale.

Gutachter:

Datum der Begutachtung: .

Patient:

Geburtsdatum:

(ID-Nr.)

Hauptdiagnose:

Asthma
COPD

\section{Arztbrief}

1.1 Zeitgerecht geschrieben (nach letzter Vorstellung)?

1.2 Diagnosen vollständig und nachvollziehbar aufgeführt?

1.3 Anamnese verständlich, ausreichend und klar?

1.4 Körperlicher Untersuchungsbefund dargestellt?

1.5 Röntgenbefund beschrieben?

1.6 Funktionsdaten (Body, Diffusion, BGA, EKG,..) sachlich verständlich und richtig dargestellt ?

1.7 Epikrise/Zusammenfassung transparent dargestellt?

1.8 Therapieempfehlung adäquat (medikamentös, nichtmedikamentös)?

1.9 Bei Therapieempfehlung wirtschaftliche Gesichtspunkte berücksichtigt

1.10 Umfang der Diagnostik angemessen?

2. Funktionsuntersuchungen

Lungenfunktion

2.1 Technische Qualität

2.2 Befundung

Sonstige Funktionsuntersuchungen

2.3 CO-Diffusionskapazität (Technische Qualität und Befundung; Mitarbeit?)

2.4 Hyperreagibilitätstest (Sollwert-, Grenzwert-Methode angegeben?)

2.5 Allergietest (Prick oder i.c.) (Protokoll vorhanden, Beurteilung adäquat?)

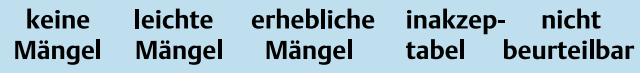

Mängel Mängel Mängel tabel beurteilbar

Bemerkungen (Kommentare bitte mit dazugehörender Fragenummer vermerken!):

\section{Ergebnisse}

\section{Alters- und Geschlechtsverteilung der Patienten}

Es wurden die Untersuchungsergebnisse, Befunde und Berichte von 396 Patienten, 218 weiblichen (55,0\%) und 178 männlichen (45,0\%) Patienten beurteilt. Die Patienten waren im Mittel 46,1 \pm 21,5 Jahre alt. Jünger als 18 Jahre waren 13,6\% der Patienten. In einem Alter von 18-40 Jahren befanden sich $24,1 \%$, zwischen 40 und 60 Jahren $28,2 \%$ und älter als 60 Jahre waren 34,6\% der Patienten.

\section{Diagnosen}

Asthma bronchiale wurde bei 166 Patienten diagnostiziert und 30 Patienten hatten eine bronchiale Hyperreagibilität. Bei 141 Patienten wurde eine COPD diagnostiziert. Bei 59 Patienten konnte keine der beiden Hauptdiagnosen festgestellt werden.
Bei 308 der über 16 Jahre alten Patienten fand sich im Mittel eine relative Sekundenkapazität $\left(\mathrm{FEV}_{1} / \mathrm{IVC}\right)$ von $72,2 \pm 15,9 \%$ und ein Atemwegswiderstand von $0,43 \pm 0,39 \mathrm{kPa} / \mathrm{l} / \mathrm{s}$. Bei den Patienten mit Asthma bronchiale fand sich bei $66,1 \%$ ein $\mathrm{FEV}_{1} \%$ des Solls von größer als $80 \%, 21,7 \%$ wiesen ein $\mathrm{FEV}_{1} \%$ Soll von $60-80 \%$ und $10,1 \%$ von $40-60 \%$ sowie $2,1 \%$ ein $\mathrm{FEV}_{1} \%$ Soll von weniger als $40 \%$ auf.

Bei den 141 COPD-Patienten hatten $20 \%$ ein $\mathrm{FEV}_{1} \%$ des Solls von größer als $80 \%$ und $33,0 \%$ ein $\mathrm{FEV}_{1} \%$ Soll von $60-80 \%$, 27,7\% zwischen 40 und $60 \%$ und $18,5 \%$ von weniger als $40 \% \mathrm{FEV}_{1} \%$ Soll. Die neu gefasste Schweregradeinteilung der GOLD-Leitlinien [7] ergibt bei den Patienten mit COPD folgende Verteilung: Stadium $0=19,2 \%$, Stadium I $=0,8 \%$, Stadium II $=21,7 \%$, Stadium III $=23,3 \%$, Stadium IV $=4,2 \% .37$ Patienten, die zwar die klinische Diagnose COPD aufwiesen, aber deren $\mathrm{FEV}_{1} / \mathrm{FVC}>70 \%$ und deren $\mathrm{FEV}_{1} \%$ des Solls $<80 \%$ waren, konnten nach GOLD gar 

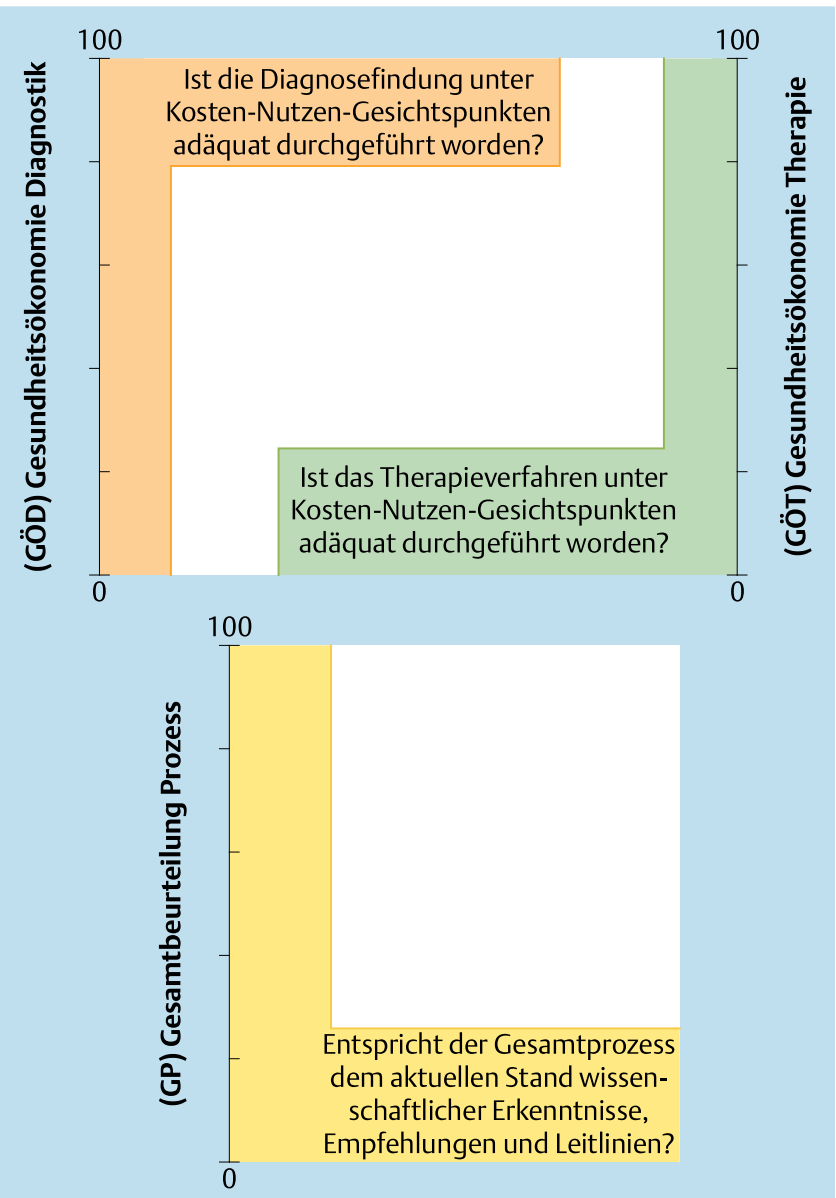

Abb. 2 Visuelle Analogskalen zur Beurteilung der gesundheitsökonomischen Aspekte von Diagnostik, von Therapie und zur Beurteilung des abgelaufenen Gesamtprozesses.

nicht klassifiziert werden [8]. Der Anteil dieser Patienten war am größten und betrug 30,8\% (Abb. 3).

\section{Bewertung der Arztbriefe}

Bei den insgesamt 1188 Beurteilungen der Briefe an den Hausarzt und der Funktionsprotokolle zeigte sich, dass mehr als die Hälfte keinen Mangel aufwiesen (Abb. 3). Allerdings ergaben sich bei fast jeder 6. Beurteilung ein „erheblicher Mangel“ oder ein als „inakzeptabel“ bezeichnetes Ergebnis. Die am häufigsten genannten Mängel bezogen sich auf die „zeitgerechte Arztbriefschreibung“ sowie die „verständliche Darstellung der Funktionsdaten“. Auch bei der „adäquaten Therapieempfehlung“ wiesen fast $20 \%$ erhebliche Mängel oder ein inakzeptables Ergebnis auf. Bei der „technischen Qualität der Lungenfunktionsdaten“ ergaben sich nur in 5,8\% der Beurteilungen erhebliche Mängel oder ein inakzeptables Ergebnis. Bei den allerdings nur in etwa einem Viertel der Fälle durchgeführten Hyperreagibilitätstests und den Allergietests ergaben sich ebenfalls erhebliche Defizite für 19,6 bzw. 21 \% der Beurteilungen. Insgesamt wurden zusätzlich von den Gutachtern 2020 persönliche, handschriftliche Bemerkungen abgegeben.

Aus den qualitativen Angaben der Gutachter wurden Qualitätsmaßzahlen errechnet, die Werte zwischen 0 und 1 annehmen konnten. Es war so eine quantitative Beurteilung der einzelnen Items der qualitätsrelevanten Prozessmerkmale der Checkliste möglich. Die Ergebnisse wurden grafisch als Rangtabelle darge- stellt und die Vergleichbarkeit der Praxen (benchmarking) ermöglicht (Abb. 4). Die Beurteilung der Wirtschaftlichkeit von Diagnostik und Therapie sowie die Beurteilung des Gesamtprozesses sind anhand einer visuellen Analogskala vorgenommen worden. Nach Auswertung durch die Projektstelle konnte auch für diese Qualitätsdimensionen ein grafisches Ranking vorgenommen werden (Abb. $\bigcirc 5$ ).

Bei willkürlicher Aufteilung der Beurteilung des Gesamtprozesses in die Gruppen von $>8$ = „gute bis sehr gute“ Prozessqualität, $6-8=$ „durchschnittliche Prozessqualität und unter $6=$ „, schwach ausgeprägte bis defizitäre“ Prozessqualität zeigt sich, dass 59,5\% der Beurteilungen eine gute bis sehr gute Prozessqualität aufwiesen, 24,1\% eine durchschnittliche und 16,4 eher schwache bzw. defizitäre Beurteilung erfuhren. Zur Klärung der Frage wurde untersucht, in wieviel Praxen Werte bezüglich des Gesamtprozesses von unter 6 vorlagen. In 39 von 44 Praxen $(88,6 \%)$ kam mindestens ein solcher Fall vor. In zwei Praxen waren mehr als die Hälfte aller Berichte mit Werten unter 6 beurteilt worden, wobei alle drei Gutachter der gleichen Meinung waren. In 5 Praxen waren mehr als 25\% der Fälle in die unterste Qualitätsstufe eingruppiert worden. Bei diesen Fällen waren dreimal alle drei Gutachter und zweimal zwei Gutachter der gleichen Meinung. Eine Häufigkeit von 10-25\% einer deutlich unterdurchschnittlichen Beurteilung kam in weiteren 13 Praxen vor. Hier waren viermal alle drei Gutachter und achtmal zwei Gutachter der Meinung, dass ein nicht ausreichender Gesamtprozess bei diesen Patienten abgelaufen ist.

\section{Diskussion}

Die Qualitätssicherung und das Qualitätsmanagement haben in den letzten 5-10 Jahren im Gesundheitswesen an Bedeutung gewonnen. Dies schlägt sich nieder in den Sozialgesetzbüchern. Die Gründe für den hohen Stellenwert, den die politische Öffentlichkeit der Qualität beimessen, hängen besonders mit den eingeschränkt vorhandenen Ressourcen zusammen [9]. Medizinische Qualität benötigt Leitlinien oder Richtlinien. Die hierdurch geschaffenen Standards sollen auf den Erkenntnissen der möglichst höchsten Stufen der evidenzbasierten Medizin beruhen [10]. Bisher gibt es diese so hochrangig nachgewiesenen Erkenntnisse aber nur in wenigen Bereichen, wie die verschiedenen Clearingberichte der ärztlichen Zentralstelle für Qualitätssicherung (ÄZQ) zu den internationalen Leitlinien zum Asthma bronchiale und zur COPD erkennen lassen [11,12,13]. Vor diesem Hintergrund und der Tatsache, dass im Bereich der klinischen und rehabilitativen Pneumologie und im Bereich der pneumologischen Schlafmedizin bereits verschiedene Peer-Review-Verfahren erfolgreich umgesetzt wurden, war es nur eine Frage der Zeit, dass auch im ambulanten Bereich ein solches Verfahren zur Qualitätssicherung eingesetzt wird. In Zeiten des vom Gesetzgeber verordneten Qualitätsmanagements hat auch die Kassenärztliche Bundesvereinigung (KBV) ein Programm zum Qualitätsmanagement entwickelt: „QEP ${ }^{\circledR}$-Qualität und Entwicklung in Praxen“ [14]. Bei diesen Programmen steht aber wegen seiner vielseitigen Einsetzbarkeit im gesamten $\mathrm{KV}$-Bereich vor allem das organisatorische Patienten- und Mitarbeitermanagement im Vordergrund. Die krankheitsspezifische Prozessqualität kann hingegen trotz eines guten Praxismanagements auf der Strecke bleiben, wenn nicht verifiziert wird, ob die abgelaufenen Prozesse wie Art der Untersuchung, Beschreibung und Interpretation der Befunde, Diagnosestellung, Therapie und erteilte Rat- 
Tab. 1 Häufigkeitsverteilung der Beurteilungskategorien für einzelne Items der Checkliste qualitätsrelevanter Prozessmerkmale

\begin{tabular}{|c|c|c|c|c|c|c|}
\hline Item & & $\begin{array}{l}\text { Anzahl der Begut- } \\
\text { achtungen (n) }\end{array}$ & $\begin{array}{l}\text { keine Mängel } \\
\text { (\%) }\end{array}$ & $\begin{array}{l}\text { leichte Mängel } \\
\text { (\%) }\end{array}$ & $\begin{array}{l}\text { erhebliche Mängel } \\
\text { (\%) }\end{array}$ & $\begin{array}{l}\text { Inakzeptabel } \\
\text { (\%) }\end{array}$ \\
\hline 1.1 & Arztbrief zeitgerecht geschrieben? & 876 & 54,9 & 20,1 & 11,5 & 13,5 \\
\hline 1.2 & Diagnose vollständig aufgeführt? & 1127 & 51,0 & 36,9 & 9,4 & 2,7 \\
\hline 1.3 & Anamnese verständlich? & 1139 & 45,3 & 36,9 & 14,0 & 3,9 \\
\hline 1.4 & $\begin{array}{l}\text { Körperlicher Untersuchungsbefund } \\
\text { dargestellt? }\end{array}$ & 1133 & 41,0 & 40,4 & 9,6 & 8,9 \\
\hline 1.5 & Röntgenbefund beschrieben? & 490 & 62,2 & 27,8 & 6,7 & 3,3 \\
\hline 1.6 & $\begin{array}{l}\text { Funktionsdaten sachlich verständ- } \\
\text { lich dargestellt? }\end{array}$ & 1144 & 48,4 & 29,5 & 18,2 & 3,9 \\
\hline 1.7 & $\begin{array}{l}\text { Epikrise/Zusammenfassung trans- } \\
\text { parent dargestellt? }\end{array}$ & 1137 & 49,4 & 33,3 & 12,2 & 5,0 \\
\hline 1.8 & Therapieempfehlung adäquat? & 1124 & 51,6 & 28,7 & 14,8 & 4,9 \\
\hline 1.9 & $\begin{array}{l}\text { Therapieempfehlung wirtschaft- } \\
\text { liche Gesichtspunkte? }\end{array}$ & 1083 & 65,7 & 22,3 & 9,1 & 2,9 \\
\hline 1.10 & $\begin{array}{l}0 \text { Umfang der Diagnostik ange- } \\
\text { messen? }\end{array}$ & 1137 & 63,5 & 24,5 & 10,2 & 1,8 \\
\hline & Lungenfunktion, techn. Qualität? & 1129 & 78,3 & 15,9 & 4,6 & 1,2 \\
\hline & Lungenfunktion, Befundung? & 1097 & 66,3 & 21,5 & 8,1 & 4,1 \\
\hline & CO-Diffusionskapazitiät? & 450 & 73,8 & 16,9 & 5,6 & 3,8 \\
\hline & Hyperreagibilitätstest? & 326 & 51,2 & 29,1 & 13,5 & 6,1 \\
\hline & Allergietest? & 351 & 53,8 & 25,1 & 8,5 & 12,5 \\
\hline
\end{tabular}

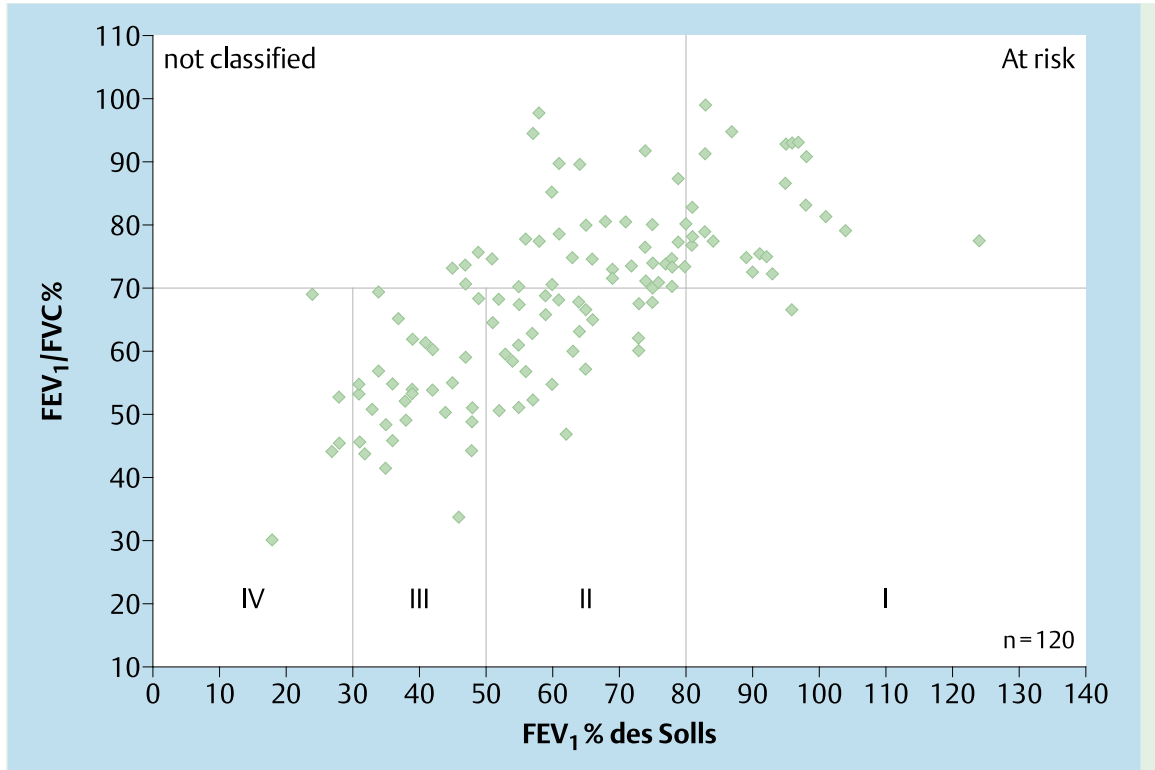

Abb. 3 Verhältnis von relativer Sekundenkapazität $\left(\mathrm{FEV}_{1} / \mathrm{FVC} \%\right)$ zu FEV ${ }_{1} \%$ Soll sowie Abgrenzung der verschiedenen Schweregrade nach GOLD bei Patienten mit COPD.

schläge wirklich so sind, wie sie den wissenschaftlichen und allgemein anerkannten Standards in der Medizin für das jeweils vorliegende Krankheitsbild entsprechen. Dies sollte aus der vom Facharzt vorgenommenen Dokumentation und aus dem Bericht für den Hausarzt hervorgehen und nachvollziehbar sein. In dem hier vorliegenden Pilotprojekt zur Sicherung der Prozessqualität in der pneumologischen Praxis haben sich 44 niedergelassene Pneumologen im norddeutschen Raum, ansässig an 11 verschiedenen Praxisstandorten bereit erklärt, sich der Beurteilung durch ihre eigenen Kollegen freiwillig zu stellen und selbst Beurteilungen der Unterlagen anderer Praxen vorzunehmen. Bei 60 angesprochenen Praxen entspricht dies einer Response-Rate von $73,3 \%$. Für die Entscheidung zur Nichtteilnahme waren überwiegend persönliche Gründe ausschlaggebend.

Die qualitätsrelevanten Prozessmerkmale sowie das Manual, in dem die Mängelkategorien dargestellt und definiert sind, wurde von einer Steuerungsgruppe der Peers entwickelt und den Teilnehmern des Pilotprojekts bekannt gemacht. Diese Methode hatte den Vorteil, dass die Standards, die nach den Kriterien des Manuals beurteilt werden sollten, einen realen Bezug zur täglichen Routine in einer pneumologischen Praxis hatten. Es ist davon auszugehen, dass hierdurch die Begutachtungsergebnisse akzeptiert werden. Durch den individuellen Ergebnisbericht wird dem Arzt dargestellt, wo Defizite in der Betreuung einzelner Patienten aufgetreten sind, die er selbst zukünftig ändern kann. Es wird ihm verdeutlicht, in welchen Bereichen andere Ärzte individuelle Schwachstellen oder auch Stärken ausgemacht haben. Der Teilnehmer erhält so die Chance, seine Kenntnisse in speziellen Bereichen seines Fachgebietes zu verbessern und sich und seine Mitarbeiterinnen und Mitarbeiter in diesen Bereichen noch intensiver fortzubilden und zu schulen. Gleichzeitig hat er die Chance durch die eigene Gutachtertätigkeit ken- 


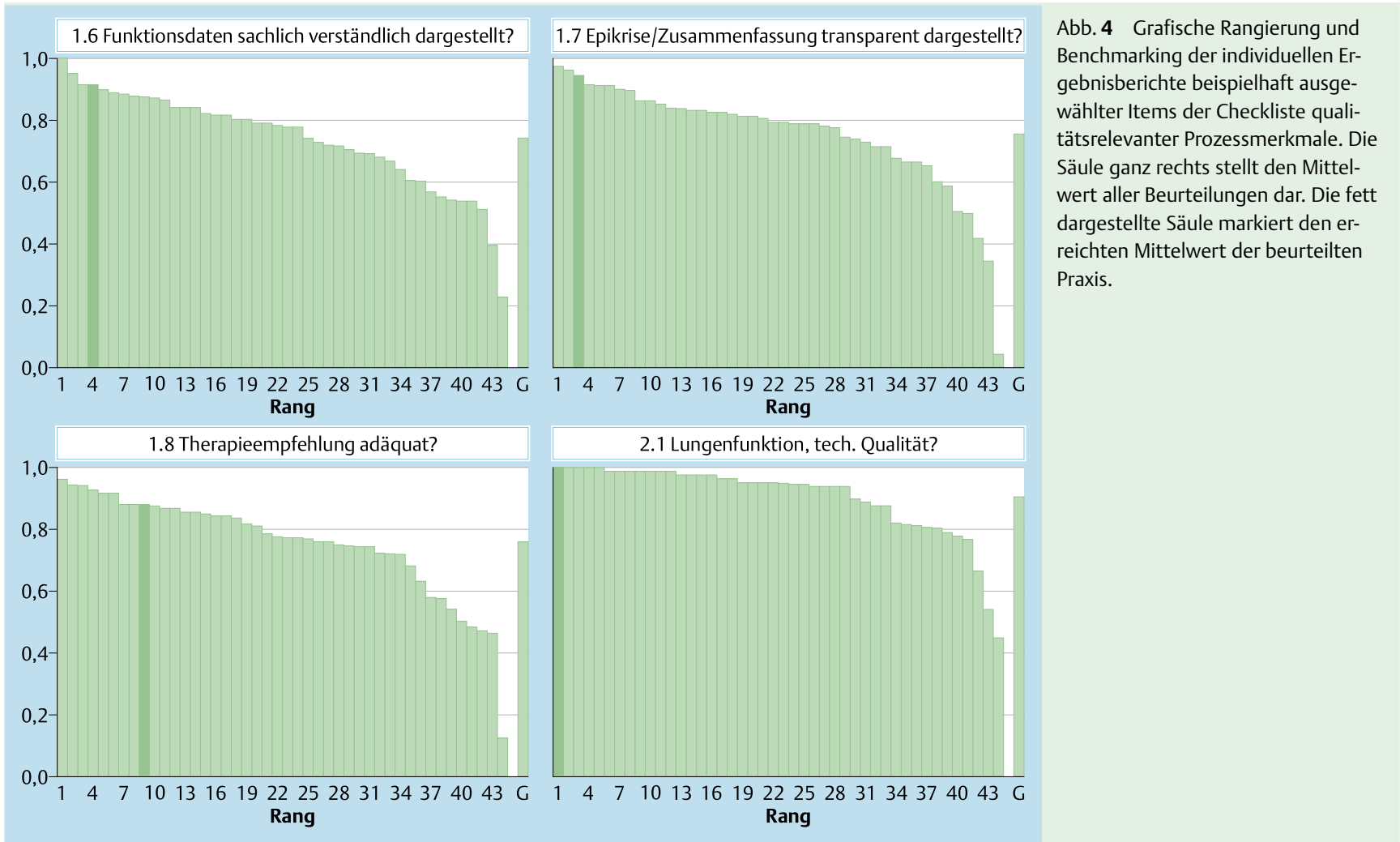

nen zu lernen, wie die Kollegen in anderen Praxen ähnliche Fälle diagnostizieren, therapieren und den Patienten bzw. den Hausarzt informieren. Dieses kann zu einer erheblichen Verbesserung seiner täglichen praktischen Tätigkeit führen.

Bei Betrachtung der Ergebnisse der Begutachtung kann insgesamt von einer überwiegend positiven Beurteilung der Prozessqualität in den pneumologischen Praxen ausgegangen werden. In verschiedenen Bereichen wurden zwar deutliche Mängel registriert, die am ehesten auf eine eher zeitbedingte oder gleichgültige Haltung bei der Dokumentation und/oder Information zurückzuführen ist. So wird angenommen, dass der weiterbehandelnde Kollege aus Zeitmangel nur Therapievorschläge liest und daher ausführliche Berichte überflüssig seien. Mit der kürzlich im Rahmen der kassenärztlichen Tätigkeit eingeführten so genannten „Pflichtberichterstattung“ fällt es aus Zeitmangel natürlich besonders schwer, für jeden Einzelfall differenzierte, präzise und auch für den Nichtspezialisten, den Hausarzt, verständliche und nachvollziehbare Berichte, die auch in ihrer Länge den ökonomischen Erfordernissen einer Praxis gerecht werden müssen, abzufassen.

Bei der Beurteilung des Gesamtprozesses durch die Peers zeigten sich bei einzelnen Fällen allerdings auch erhebliche Defizite. In 20 Praxen lagen 10\% der Beurteilungen unter einem Wert von 6 auf der visuellen Analogskala. In 95\% dieser Beurteilungen waren mindestens 2 oder sogar alle 3 Peers der Meinung, dass der Gesamtprozess bei diesen Fällen nicht ausreichend abgelaufen war. Dies ist ein Hinweis für eine gute Übereinstimmung der Meinung der Peers zumindest in der Beurteilung der im Gesamtprozess unterdurchschnittlich abschneidenden Fälle. Weiterhin weist es daraufhin, dass in einem beachtlichen Teil der Fälle die Gutachter nicht mit dem abgelaufenen Prozess zufrieden gestellt werden konnten und dass hier Verbesserungspotenzial besteht. Dabei darf nicht vergessen werden, dass die Praxen, die sich die Mühe gemacht haben an dem Projekt teilzunehmen, sicher eine Positivselektion darstellen.

Die Güte von Beurteilungsübereinstimmungen hängt auch wesentlich von der Art der Erkrankung ab und der hieraus resultierenden Möglichkeit in entsprechenden Leitlinien klare und scharf umschriebene Definitionen bestimmter Zustände zu geben. So fanden Hofer u. Mitarb. [15] eine deutlich höhere Reliabilität bei der Begutachtung von Patienten mit Diabetes mellitus und arterieller Hypertonie als bei Patienten mit COPD oder verschiedenen akuten Erkrankungen. Untersuchungen zur Intraund Interraterreliabilität einzelner Items einer Checkliste mit einem modifizierten Kappa-Koeffizienten haben bei der Sicherung der Prozessqualität in den Schlaflaboren gezeigt, dass bei sehr allgemein und wenig differenziert beschreibbaren Items die Reliabilität gering war [16]. Dies betraf dort z. B. die Items „adäquate Diagnostik Begleiterkrankungen“ oder „,adäquate Therapie Begleiterkrankungen“. Solche Items lassen den Peers einen relativ großen Spielraum bezüglich der Bewertung der jeweils vorliegenden Unterlagen mit dem Resultat einer hohen Variabilität und damit geringen Interrater-Reliabilität.

Zurzeit lässt sich noch nicht feststellen, inwieweit von den Teilnehmern an der Sicherung der Prozessqualität Konsequenzen für ihre tägliche praktische Arbeit aus ihrem persönlichen Ergebnisbericht gezogen werden. Die Arbeitshypothese für eine Wiederholung des Pilotprojektes lautet, dass es zu einer deutlichen und signifikanten Verbesserung in der Prozessqualität kommen wird. Es muss aber auch davon ausgegangen werden, dass einzelne Teilnehmer den organisatorischen Aufwand einer kontinuierlichen Teilnahme scheuen werden. Von den anfänglich 12 Teilnehmern der ersten Pilotstudie 2003/2004 haben an der 2. Pilotstudie 2004/2005 10 Praxen erneut teilgenommen. Aus einer Gemeinschaftspraxis hatten bei der Wiederholungsstudie beide Partner mit eigenen Patienten teilgenommen, sodass die Wiederholerrate zwischen $83 \%$ und $92 \%$ anzusetzen 


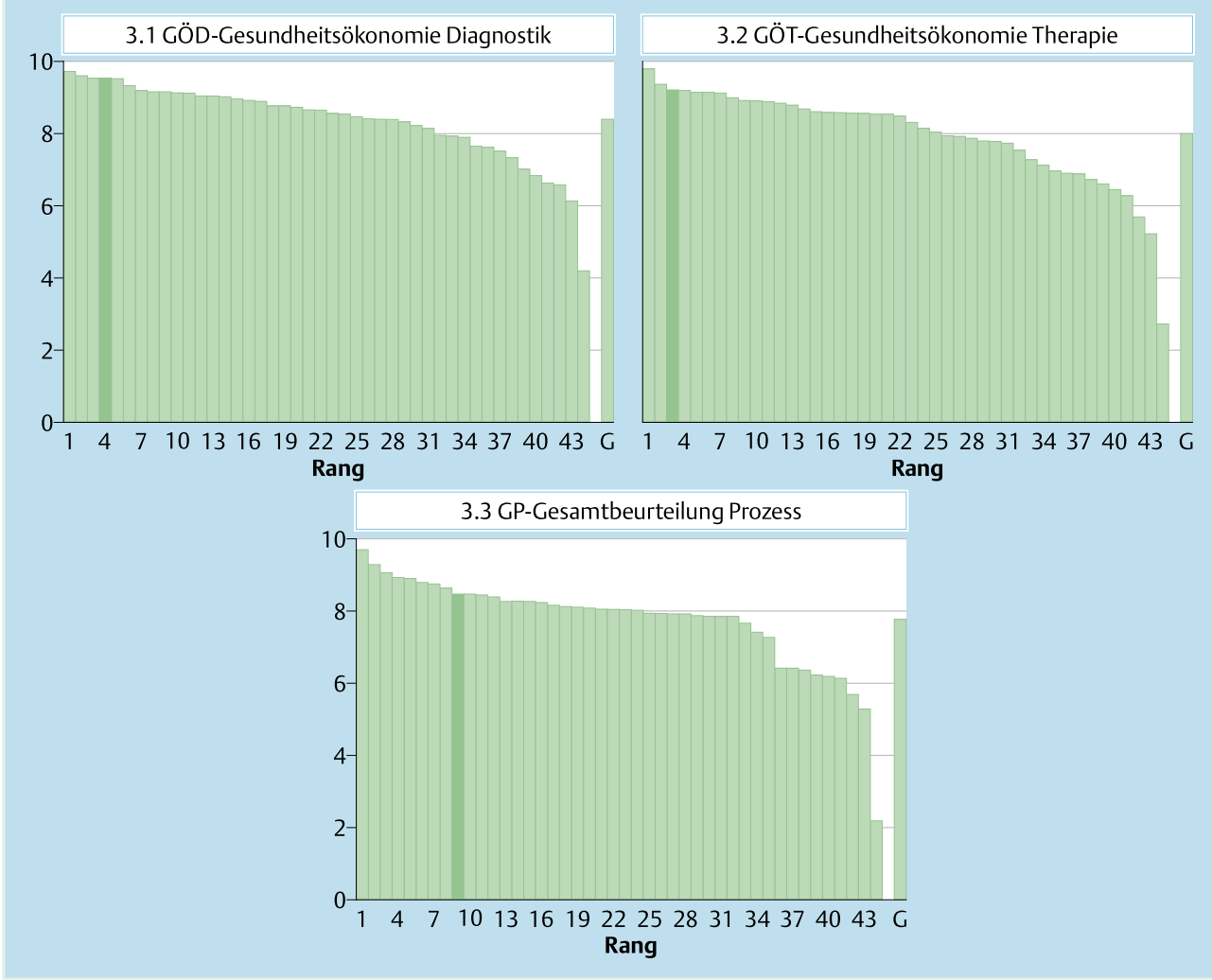

Abb. 5 Grafische Rangierung und Benchmarking der individuellen $\mathrm{Er}$ gebnisberichte der visuellen Analogskalen zur gesundheitsökonomischen Bewertung der Diagnostik, der Therapie und zur Bewertung des abgelaufenen Gesamtprozesses. Die Säule ganz rechts stellt den Mittelwert aller Beurteilungen dar. Die fett dargestellte Säule markiert den erreichten Mittelwert der beurteilten Praxis.

ist. Für die an diesem Pilotprojekt teilnehmenden Praxen ergab sich eine Wiederholerrate von ebenfalls $82 \%$, was als Ausdruck einer hohen Akzeptanz der Methode gewertet werden kann. Erste Auswertungen weisen auf eine signifikante Verbesserung der Prozess- und Ergebnisqualität hin. Einzelheiten hierzu werden in einer weiteren Publikation dargestellt.

Die mit diesem Pilotprojekt gestellten Ziele und Fragen lassen sich so beantworten, dass in der pneumologischen Facharztpraxis die Beurteilung und die Sicherung der Prozessqualität grundsätzlich möglich ist. Anhand der aus dieser Pilotstudie gewonnenen Erkenntnisse erscheint sie unbedingt erforderlich und sinnvoll zur Verbesserung der Patientenbehandlung, bedingt auch durch eine Verbesserung der Arzt-Arzt-Information. Das PeerReview-Verfahren hat sich bisher als Beurteilungsverfahren bewährt. Anhand vieler persönlicher Äußerungen war es vor allem der Erfahrungsgewinn, wie läuft bei anderen Kollegen der Prozess tatsächlich ab und wie beurteilen andere Kollegen meinen Prozessablauf, der den erforderlichen zeitlichen Aufwand rechtfertigt. Eine wichtige Voraussetzung für den reibungslosen Ablauf eines Peer-Review-Verfahrens ist die Termintreue der Teilnehmer insbesondere bei der Bereitstellung der zu begutachtenden Unterlagen und der Durchführung der Begutachtung. Nachlässigkeiten in diesem Bereich bei einzelnen Gutachtern können sehr schnell zu einer enormen zeitlichen Gesamtverzögerung des Projektes führen. Bei der Peer-Review-Begutachtung pneumologischer Kliniken ist man daher dazu übergegangen, Verträge mit den Gutachtern abzuschließen und Vertragsstrafen für Zeitversäumnisse zu vereinbaren.

Aufgrund der bisher guten Erfahrungen in beiden Pilotprojekten ist daran gedacht, dieses System über den regionalen Bereich hinaus einzusetzen und den Kollegen die Teilnahme an einer Sicherung der Prozessqualität in der pneumologischen Praxis mit dem Peer-Review-Verfahren auch bundesweit zu ermöglichen. Weitere Untersuchungen werden darüber Auskunft geben in- wieweit durch regelmäßige Wiederholung dieser Qualitätssicherungsmaßnahme eine kontinuierliche Sicherung und Verbesserung der Qualität möglich ist.

\section{Anmerkung}

Diese Arbeit entstand unter wesentlicher Mithilfe der niedergelassenen Lungenärzte:

J. Becker, J. Grützmacher, H. ten Hoff, H. Leiner, K. Löck, R. Örtel, H. Schwarting, H. Timmermann und der Med. Dok. Ass. Susanne Möbus, Norderney.

Unser Dank gilt auch den übrigen teilnehmenden Lungenärzten und für die Unterstützung den Firmen Boehringer-Ingelheim, Ingelheim und Pfizer, Karlsruhe.

\section{Literatur}

1 Penzel T, Hein H, Rasche Ket al: Leitfaden für die Akkreditierung von Schlafmedizinischen Zentren der Deutschen Gesellschaft für Schlafforschung und Schlafmedizin (DGSM). Somnologie 2000; 4: 181-187

2 Fischer J, Raschke F, Kutschmann M: Die Checkliste qualitätsrelevanter Prozessmerkmale der Deutschen Gesellschaft für Schlafforschung und Schlafmedizin (DGSM) zur Sicherung der Prozessqualität im akkreditierten Schlaflabor. Somnologie 1999; 3: 335 - 346

3 Penzel T, Berger M, Clarenbach Pet al: H. Zur Qualitätskontrolle von Schlaflabors in der Bundesrepublik Deutschland. Wien Med Wschr 1999; (Sonderheft): $120-124$

4 Fischer J, Mayer G, Peter JHet al: Nicht-erholsamer Schlaf. Leitlinie S2 der Deutschen Gesellschaft für Schlafforschung und Schlafmedizin. Berlin, Wien: Blackwell 2002

5 Jäckel WH, Maier-Riehle B, Protz Wet al: Ein Verfahren zur Analyse der Prozessqualität stationärer Rehabilitationsmaßnahmen. Die Rehabilitation 1997; 36: 224-232

6 Köhler D, Goeckenjan G, Rünz J: Evolutionäre Qualitätssicherung - Ein neues Konzept zur Verbesserung der Prozess- und Ergebnisqualität. Med Klin 1998; 93: 191 - 196 
7 Fabbri LM, Hurd SSet al: for the GOLD Scientific Committee. Global Strategy for the Diagnosis, Management and Prevention of COPD: 2003 Update. Eur Respir J 2003; 22: 1 -1

8 Köhler D, Fischer J, Raschke Fet al: Usefullness of GOLD classification of COPD severity. Thorax 2003; 58: 825-825

9 Lauterbach KW, Schrappe M: Gesundheitsökonomie, Qualitätsmanagement Evidence based Medicine. Eine systematische Einführung. Stuttgart: Schattauer 2001

10 Deutsche Gesellschaft für Pneumologie: Empfehlungen zur Qualitätssicherung in der ambulanten und stationären pneumologischen Versorgung. Pneumologie 1997; 51: 569-574

11 Zentralstelle der Deutschen Ärzteschaft zur Qualitätssicherung in der Medizin: Leitlinien - Clearing-Bericht „Asthma bronchiale“. München, Wien, New York: Zuckschwerdt 2001
12 Zentralstelle der Deutschen Ärzteschaft zur Qualitätssicherung in der Medizin: Leitlinien - Clearing-Bericht „COPD“. äzq Schriftenreihe, Bd. 142003

13 Franzen D, Fessler J, Fischer Jet al: Das Leitlinien Clearingverfahren COPD. Empfehlungen für eine nationale Leitlinie. Pneumologie 2004; 58: $858-862$

14 Kassenärztliche Bundesvereinigung: „QEP ${ }^{\circledR}$ - Qualität und Entwicklung in der Praxis“. www.kbv.de/qm

15 Hofer TP, Asch SM, Hayward RAet al: Profiling quality of care: Is there a role for peer review? BMC Health Serv Res 2004; 4: 9-9

16 Kutschmann M, Raschke F, Fischer J: Verbesserung der Prozessqualität in der Schlafmedizin durch ein Peer-Review-Verfahren. Somnologie 2002; 6: $85-92$ 